\title{
Frontières
}

\section{L'infirmière et le soulagement de la douleur}

\section{La douleur, le lien qui unit les infirmières de tous les milieux}

\section{France Hudon}

Volume 17, numéro 2, printemps 2005

Surtout, ne pas souffrir

URI : https://id.erudit.org/iderudit/1073494ar

DOI : https://doi.org/10.7202/1073494ar

Aller au sommaire du numéro

Éditeur(s)

Université du Québec à Montréal

ISSN

1180-3479 (imprimé)

1916-0976 (numérique)

Découvrir la revue

Citer cet article

Hudon, F. (2005). L'infirmière et le soulagement de la douleur : la douleur, le lien qui unit les infirmières de tous les milieux. Frontières, 17(2), 86-88.

https://doi.org/10.7202/1073494ar
Résumé de l'article

L'infirmière est souvent intervenante de première ligne dans toutes les démarches concernant le dépistage, l'évaluation, la recherche et l'application d'un traitement, l'enseignement aux patients et la réévaluation du symptôme de la douleur. Pour accomplir ces tâches, elle doit maintenir à jour ses connaissances et accepter de jouer un rôle important au sein de l'équipe multidisciplinaire. 


\section{Résumé}

L'infirmière est souvent intervenante de première ligne dans toutes les démarches concernant le dépistage, l'évaluation, la recherche et l'application d'un traitement, l'enseignement aux patients et la réévaluation du symptôme de la douleur. Pour accomplir ces tâches, elle doit maintenir à jour ses connaissances et accepter de jouer un rôle important au sein de l'équipe multidisciplinaire.

Mots clés: soulagement de la douleurrôle infirmier.

\section{Abstract}

The nurse is often the front contributor in all approaches regarding tracking, evaluation, research and administration of a treatment, as well as patients' education and revaluation of pain symptoms. In order to perform, the nurse must enhance her/his knowledge and accept to act her/ his role into the multidisciplinary team.

Keyswords: pain management nursing role.

\section{L'INFIRMIÈRE ET LE SOULAGEMENT DE LADOULEUR La douleur, le lien qui unit les infirmières de tous les milieux}

\section{France Hudon, \\ chargée de cours en sciences infirmières, Université de Montréal et infirmière en oncologie et en soins palliatifs.}

Il est impossible pour une infirmière d'échapper au contact de la douleur. On la rencontre partout, de la salle d'accouchement à la pouponnière, à l'urgence, aux soins intensifs, aux soins palliatifs et à domicile; peu importe, elle est là, fidèle compagne de la journée de travail, avec à ses côtés sa sœur jumelle, la souffrance, celle qui touche non seulement le corps mais atteint l'âme et l'enchaîne. C'est très souvent pour la soulager que l'infirmière choisit cette profession, car rien n'est plus difficile, douloureux que d'assister impuissant à la souffrance de l'autre et si on finit par accepter l'inévitable passage de la mort, jamais on ne s'habitue à la présence de la douleur. Le besoin de lutter contre elle est la motivation profonde de la profession infirmière, et ce bien souvent avant le désir ou le besoin de guérir le malade. Alors, si la douleur est aussi présente dans le travail de l'infirmière et son désir de la soulager si important, que peut-elle faire pour le malade en proie à la souffrance? À quel moment peut-elle intervenir et comment est-elle préparée à ce rôle de soignante de la douleur? Qui peut la soutenir dans sa démarche?

\section{LE RÔLE DE L'INFIRMIÈRE DANS LE SOULAGEMENT DE LA DOULEUR}

La souffrance, qui inclut la douleur physique mais aussi la souffrance morale, le déséquilibre du cœur et les bouleversements de la vie est un mystère pour l'Autre. Cet étranger que je suis m'interdit toute véritable connaissance de la souffrance du malade. Je ne suis que le témoin de cette douleur et rien ne pourra jamais me donner la certitude que je sais ce que l'autre vit, car toute connaissance véritable passe par l'expérience, et même l'expérience de ce qui m'apparaîtrait avoir été une douleur semblable à celle vécue par mon patient ne pourrait être qu'une approximation teintée de ma couleur, de mes sens, de mon expérience et par le fait même unique et propre à moi-même. Je ne saurai jamais ce que l'autre a vécu. Pour soulager la douleur, ce fait incontournable doit être immédiatement compris et accepté. «La douleur est tout ce que la personne qui souffre décrit comme telle, elle existe chaque fois que la personne qui l'éprouve le dit (McCaffery, 1979)». Je ne sais pas ce que l'autre éprouve, lui seul peut me le dire et je ne peux mettre en doute sa parole. Le malade doit dévoiler lui-même le mystère de sa douleur et l'infirmière est en première ligne pour lui permettre de le faire. 


\section{LE DÉPISTAGE, \\ LA PREMIĖRE ÉTAPE \\ DANS LE SOULAGEMENT \\ DE LA DOULEUR}

Ouvrir d'emblée la porte au malade, questionner systématiquement la douleur par un: «Avez-vous mal? » répété à chaque rencontre; en faire, selon la suggestion de l'International Association of Study of Pain (IASP) «le cinquième signe vital », voilà la première et indispensable étape qui fera en sorte que l'énigme commencera peu à peu à se dévoiler. Questionner et accepter sans restriction la réponse donne au malade le droit de dire sa douleur et par le fait même lui accorde l'espoir d'être soulagé. Mais que faire pour le bébé, le malade qui ne peut répondre, la personne atteinte de déficits cognitifs? Alors il faut bien observer ses comportements, questionner ses proches, ses soignants, utiliser les outils d'évaluation comportementale et, dans le doute, agir, surtout ne pas lui refuser le soulagement à cause de notre incapacité à être rassuré par son aveu de douleur.

\section{ÉVALUER LA DOULEUR: LA RESPONSABILITÉ NON SEULEMENT DU MÉDECIN MAIS DE TOUTES LES INFIRMIÈRES AU CHEVET DU PATIENT}

Si demander au malade de dire sa douleur est important, cette première étape en révèle cependant bien peu sur le problème que le soignant aura à résoudre et il faut aborder immédiatement la seconde étape de l'évaluation approfondie du mal qui afflige la personne. L'infirmière, en mentionnant simplement au médecin: «Docteur, votre patient est très souffrant, pouvez-vous le soulager? ", fournit une information très incomplète qui ne permet pas au médecin de prescrire un analgésique ou un co-analgésique approprié. Seule une évaluation systématique faite à l'aide d'outils validés permet de donner l'information nécessaire à toute prescription antalgique. Cette évaluation dépasse largement le cadre de la fameuse "échelle de douleur». Bien que celle-ci apporte un peu de lumière sur ce que pense le malade de sa douleur et puisse devenir par la suite un excellent guide pour vérifier l'efficacité des traitements entrepris, elle n'éclaire pas sur les causes, les caractéristiques et la façon de soulager la douleur. L'évaluation approfondie permet aussi de juger de l'urgence de la situation et de mettre rapidement en place les traitements nécessaires.

Linfirmière de chevet est la mieux placée pour faire cette évaluation et procéder à l'examen physique du patient. Malheureusement, elle est souvent peu préparée à jouer ce rôle et ce manque de connaissances risque d'entraîner des délais dans l'obten-

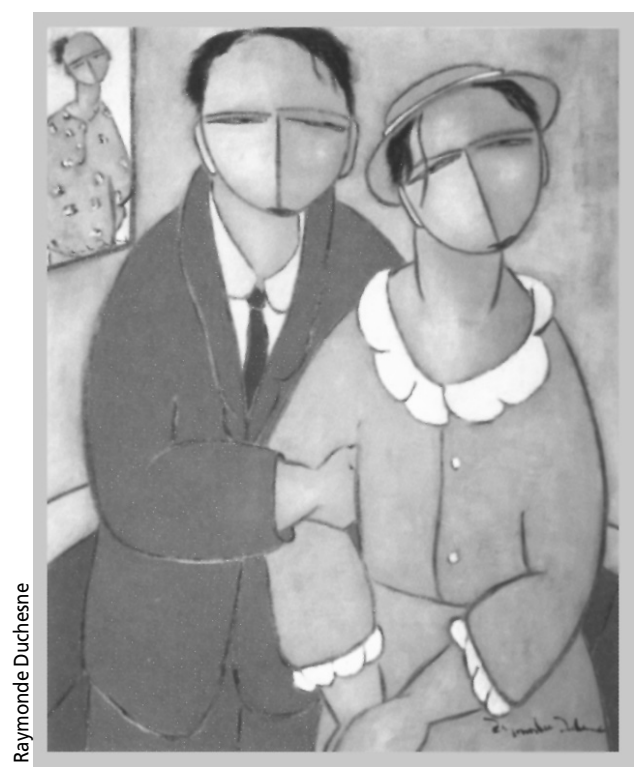

relief du temps passé

tantôt désordre dans les sentiers

tantôt sagesse de l'aurore

là jongle la vie

tion d'une prescription adéquate lorsque le médecin ne peut être lui-même présent auprès du malade. Ma pratique et l'observation de celle de mes confrères et consœurs m'a permis de constater que, très souvent, il devient beaucoup plus simple d'obtenir une prescription analgésique adéquate par téléphone, lorsque le portrait de la douleur présenté au médecin est complet et comporte les éléments nécessaires à sa compréhension de la situation et lui donne le droit moral et légal de prescrire.

\section{LE TRAITEMENT DE LA DOULEUR}

Il existe un très large éventail de médicaments analgésiques et co-analgésiques ainsi que des approches non médicamenteuses pour arrêter partiellement ou complètement le processus douloureux. Ce sont les réponses du patient au moment de l'évaluation et son examen physique qui détermineront quelles approches il est préférable d'utiliser. C'est au médecin de décider de la prescription et, fréquemment, ce sera à l'infirmière de l'administrer. Le soulagement de la douleur implique très souvent l'utilisation d'analgésiques puissants qui présentent de nombreux effets secondaires et un potentiel de risque élevé pour les patients. Le code de déontologie des infirmières stipule que: «L'administration sécuritaire d'un médicament nécessite que l'infirmière connaisse la classe du médicament, son mécanisme d'action, les principaux effets secondaires, les réactions thérapeutiques, les condi- tions d'administration, les doses usuelles, les contre-indications et les interactions médicamenteuses» (Perspective infirmière, nov. 2004). Le problème actuel est que très peu de formation sur la douleur est offerte aux étudiantes en nursing, que ce soit au cégep ou à l'université. Les infirmières au cégep reçoivent en moyenne 3 à 7 heures de cours sur le sujet de la douleur; l'Université de Montréal offre un cours de 45 heures à l'éducation permanente, mais ce cours n'est pas obligatoire.

Les infirmières ayant obtenu leur diplôme il y a un certain nombre d'années déjà ne peuvent non plus avoir été formées adéquatement sur le sujet. Les connaissances évoluent très rapidement et des recyclages annuels sont obligatoires pour avoir les connaissances nécessaires pour remplir les obligations liées au Code de déontologie. Les institutions de soins n'offrent pas non plus un nombre d'heures de formation suffisant à toutes les infirmières pour remplir cette obligation. Ceci est un problème majeur, car les erreurs d'administration médicamenteuses concernant les analgésiques puissants se soldent fréquemment par le décès du patient.

Par exemple, sur 276 erreurs d'administration médicamenteuse ayant été rapportées à l'organisme américain JAHCO (Joint Commission on Accreditation of Healthcare Organizations), 21\% impliquaient des opioïdes. Cette catégorie était la plus importante parmi celles qui avaient été signalées. La quasi-totalité des accidents rapportés avec cette classe thérapeutique $(98 \%)$ a entraîné la mort du patient (Pradines, 2004).

\section{L'ENSEIGNEMENT}

\section{AU PATIENT ET AUX PROCHES}

Tous les efforts fournis par l'équipe soignante pour évaluer la situation et instaurer un traitement adéquat pour le soulagement de la douleur risquent d'être perdus si l'on ne peut obtenir une collaboration entière du malade et de ses proches. De nombreuses craintes, des mythes tenaces, entourent l'utilisation de la médication analgésique et même le fait de parler de la douleur et la demande de soulagement peuvent être perçus par certains patients (et malheureusement par certains soignants) comme étant un signe de faiblesse. Il faut parfois travailler très fort pour arriver à faire accepter une prescription de morphine tant les fausses croyances entourant cette médication sont nombreuses et tenaces. L'infirmière doit alors rassurer le malade et enseigner les bienfaits de l'utilisation analgésique. Elle devra aussi expliquer les principes de l'utilisation sécuritaire de la médication et surtout les nombreux effets secondaires qui, lorsqu'ils sont insuffisamment traités, 
risquent de compromettre sérieusement le démarche de soins. Cet enseignement est absolument essentiel, particulièrement avec la douleur chronique, car la personne doit alors prendre en charge l'administration de sa médication, et ce pendant des mois, sinon de nombreuses années. Les aspects psychosociaux de la douleur et de son traitement doivent aussi être expliqués et les nombreuses approches complémentaires intégrées, car le succès du soulagement de la douleur survient rarement par la seule administration d'une thérapie médicamenteuse.

\section{LA RÉÉVALUATION DE LA DOULEUR}

Lénigme constituée par la douleur et son soulagement ne peut se résoudre que par des essais (et par le fait même des erreurs); seule la réponse du patient pourra dire si le choix fait était le bon. Cette dernière étape dans l'organisation du suivi de la douleur est donc capitale et l'infirmière y joue un rôle de premier plan. L'hésitation de plusieurs médecins, ayant les connaissances nécessaires, à prescrire une analgésie plus puissante tient souvent à leur manque de temps pour offrir un suivi étroit au patient et à la crainte de lui causer un tort par l'absence de supervision nécessaire après la prescription. Dre Johanne Desforges, médecin très impliquée dans le soulagement de la douleur chronique et ayant une pratique de bureau privé importante, me confiait récemment qu'elle pourrait personnellement soulager beaucoup plus de patients présentant des douleurs sévères si elle avait plus de temps pour en faire le suivi. Elle souhaiterait travailler en étroite collaboration avec une infirmière avec qui elle pourrait partager les tâches d'évaluation, d'enseignement et de réévaluation des patients.

Dre Desforges n'est certainement pas la seule médecin à penser ainsi et nous pouvons entrevoir ici le rôle important que les infirmières peuvent jouer dans le suivi des patients en clinique privée.

Revoir la personne rapidement après une prescription analgésique, vérifier la réponse au traitement, revoir les effets secondaires, ajuster la médication lorsque nécessaire sont des étapes obligatoires de la thérapie. La pratique actuelle cependant tient rarement compte de cette nécessité et de nombreux patients reçoivent leur médication de façon routinière, sans que l'on ait le souci de refaire avec eux le questionnaire d'évaluation de leur douleur. On voit ainsi des prescriptions d'analgésiques d'un dosage nettement insuffisant ou carrément inefficace administrées pendant parfois des mois. J'ai recueilli personnellement le témoignage de plusieurs infirmières œuvrant en centre de soins de longue durée reconnaissant administrer de l'acétaminophène régulièrement à des personnes âgées souffrant d'arthrite sévère pendant des mois et même des années sans remarquer d'amélioration de la condition du patient.

Cet état de fait est inacceptable et ne peut être corrigé que par l'implication des infirmières dans la réévaluation de la douleur et leur souci de demander au médecin une modification de l'ordonnance lorsque cela s'avère nécessaire.

\section{LA DOULEUR CHRONIQUE UNE MALADIE FRÉQUENTE}

Lorsqu'elle devient chronique, la douleur n'est plus un signal d'alarme, elle devient alors une véritable maladie qu'il faut traiter rapidement, car elle a des conséquences très sérieuses sur la qualité de vie des personnes atteintes. Malheureusement, comme toute maladie chronique, son traitement demande une approche élargie, qui demande la participation d'une équipe de soins non seulement dévouée et disponible mais ayant acquis des connaissances spécifiques à cette problématique et ayant les moyens nécessaires pour mettre en place les traitements appropriés. Au Québec, il existe présentement 12 centres offrant ce type de clinique spécialisée en douleur, ce qui est très peu si l'on tient compte que la recherche sur la prévalence de la douleur chronique au Canada (Moulin et al., 2002) a démontré qu'environ $30 \%$ de la population active est aux prises avec une douleur chronique non cancéreuse. Le délai d'attente pour être vu dans ces cliniques est de plusieurs mois et parfois jusqu'à un an. Fait intéressant, quelques-unes de ces cliniques ne comptent pas d'infirmière dans leurs effectifs, ce qui pourrait pourtant alléger considérablement la tâche des médecins et leur permettre de voir un plus grand nombre de patients.

\section{INFIRMIÈRE CLINICIENNE DE LA DOULEUR: UN RÔLE EN OR POUR LES INFIRMIÈRES}

Par sa place en première ligne auprès des patients, l'infirmière est une personne clé pour collaborer au soulagement de la douleur des patients. La possibilité qu'offre la loi 90 d'élargir le champ de pratique des différents professionnels de la santé ouvre une grande porte pour permettre l'instauration d'un rôle d'infirmière clinicienne en douleur. Ce rôle pourrait s'exercer dans presque tous les milieux de soins, puisque, comme nous l'avons déjà dit, la douleur est omniprésente et représente un défi journalier pour toutes les équipes soignantes. Mais pour que ces rôles soient créés et reconnus dans toute leur valeur, il est urgent que la formation infirmière rehausse le niveau de connaissances en douleur pour toutes les infirmières, avec des possibilités de perfectionnement d'un niveau universitaire avancé pour que les infirmières intéressées à une pratique autonome puissent prendre les responsabilités élargies inhérentes à leur travail.

\section{SOULAGER LA DOULEUR: UN DÉFI} POUR TOUS LES INTERVENANTS

La médecine d'aujourd'hui a fait d'énormes progrès et la compréhension des causes physiologiques et des aspects psychosociaux impliqués dans le phénomène de la douleur sont maintenant connus par la communauté scientifique. Plusieurs approches médicamenteuses et non médicamenteuses ont fait leurs preuves et il est possible pour les malades d'espérer une amélioration importante de leurs souffrances. C'est pourquoi il est urgent que les médecins, les infirmières, les pharmaciens, mais aussi tous les intervenants de l'équipe soignante (préposé aux bénéficiaires, travailleur social, physiothérapeute, psychologue, pour n'en nommer que quelques-uns) s'approprient ces connaissances et déploient tous leurs efforts pour soulager les malades.

Ce n'est que par la collaboration de tous les professionnels de la santé, avec une reconnaissance et une acceptation inconditionnelle du rôle de chacun, que l'on peut espérer arriver à un réel soulagement de la douleur.

Je termine sur cette citation de Simone Weil:«Il n'y a ici-bas que la douleur physique et rien d'autre qui ait la propriété d'enchaîner la pensée; à condition qu'on assimile à la douleur physique certains phénomènes difficiles à décrire, mais corporels, qui lui sont rigoureusement équivalents. L'appréhension de la douleur physique notamment est de cette espèce» (Weil, 1966, p. 99-100). Ceci résume bien selon moi l'importance de traiter envers et contre tout la douleur physique.

\section{Bibliographie}

MOULIN, D.E., A.J. CLARK, M. SPEECHLEY, P.K. MORLEY-FORSTER (2002). "Chronic pain in Canada-prevalence, treatment, impact and the role of opioid analgesia», dans Pain Res. Manag., hiver, 7(4), p. 179-84.

PERSPECTIVE INFIRMIÈRE (2004). «L'administration de médicaments: rappel des obligations déontologiques», Le Journal, supplément de Perspective infirmière, novembredécembre, vol. 2, n 2 , p. 6 .

PRADINES, Bernard (2004). «Prescriptions antalgiques anticipées », <http://www.geocities.com/bpradines/Douleuranticipation. $\mathrm{htm}>$.

WEIL, Simone (1966). Attente de Dieu, Paris, Éditions Fayard. 